\title{
Design of an Effective Assessment-Feedback Scheme through a Virtual Learning Environment
}

\author{
Héctor Jiménez-Salazar, Tiburcio Moreno-Olivos, Alfredo Mateos-Papis \\ Universidad Autónoma Metropolitana, Unidad Cuajimalpa \\ $\{$ hjimenez, tmoreno, amateos\}@correo.cua.uam.mx
}

\begin{abstract}
The teaching-learning process faces big challenges to incorporate information and communication technologies (ICTs) in an effective way. Considering virtual learning environments (VLEs) which provide higher-education students with the possibility to solve assessments focused on solving problems with multiple-choice questions (MCQ), and which offer the student feedback in relation to the answers, this paper presents a proposal to design the medular part of such kind of VLEs: an assessment-feedback scheme. This proposal considers the fundamentals and the results of various works on feedback and on learning-processes. Additionally, the scheme should capture the student's browsing for further analysis.
\end{abstract}

Keywords: feedback, assessment, virtual learning environment.

\section{Introduction}

From the seminal work of Sadler [12] to date, several papers have been published, which analyze the topic of feedback in assessment, for example papers about: sustainable feedback, feedback models for learning $[12,13,6]$; formative assessment and feedback, auto-regulated learning $[2,3,9,10,16]$; perceptions on the feedback process, practice development of sustainable feedback [15, 4], among others. Also, the use of ICTs on educative processes has allowed the emergence of Learning Management Systems (LMS) or VLEs, and this has powered electronic assessment (e-assessment) and feedback in e-assessment. Several initiatives have emerged to find/create better ways to use ICTs in the teaching-learning process. For example, concerning feedback, some works try to capture the perception of students [1], or personalize the LMS according to the user [11], or analyze the recorded behavior of students when using a LMS, through Weka [14].

The problem that this article addresses is the lack of knowledge, that the authors have perceived, about the effectiveness of the feedback that students receive when they learn a subject though the use of exercises.

In order to study this problem, this article proposes the use of a VLE feedback scheme, as an effective form, not only to analyze the way in which students request feedback when they solve problems, but also to serve as a support tool to help students with their learning process. This scheme should explore the relations among three incidental factors on feedback: 1) The depth 
of understanding that the student has when facing a problem, 2) If the problem statement is clear to the student, and 3) The level of certainty that the student has when solving a problem (an exercise).

The remaining sections of this article are organized as follows. Section 2 offers a survey on the relevance of feedback in the learning process, which is remarked by seven principles [9] of good feedback practices in self-assessment, and which form the basis of the proposal of his article. Section 3 presents a brief summary about some implementations of feedback using VLE, which can contrast with the present work. Section 4 presents the aforementioned proposal. The closing section presents the conclusions of this work.

\section{Background}

An effective feedback, periodically delivered, may help students to know how well they are doing their work. Feedback may help them decide how to be more effective or how to enhance the management of their workload, and at the ending, be satisfied with their learning process.

When a student lacks prior knowledge, necessary to be able to learn a subject, feedback may become a very useful tool. Currently, the increasing number of higher education students that a professor has to attend prevents face-to-face feedback with all students. In such conditions, ICTs can become an important resource to fulfill the purpose of feedback.

A common situation in the progress of a course is to leave feedback for later, or even to postpone it to the end of the course not giving students the opportunity to learn from their mistakes, and without allowing them to take advantage of feedback in the subsequent assessments of the course [16], [5].

It has been found that the remarks of low-value feedback focus on issues related to language (grammar, punctuation, etc.), factual accuracy or bibliographic finesse. This type of feedback is mostly cryptic; use marks on the edge of the sheet, punctuation marks, or imperatives and affirmations (such as "explain") (Lea \& Street 2000 cited in [6]). Besides, the students can only get an incomplete or uncertain understanding ([6]). Research on student's perceptions and experiences on feedback comments reveals a similar status.

It is difficult for professors to offer feedback, particularly due to the unfavorable existing conditions to help students, for example, feedback requires a lot of, unavailable, dedication time. Nevertheless, the effect of feedback can benefit, in a significant way, by getting good and timely information to the students, and verifying that they use that information when doing their work.

\section{Related Work}

This section presents published ideas that deal with feedback, from the point of view of ICT.

In [11] the authors propose an online framework to help in the preparation of a selected LMS so that it can automatically detect the learning style of its 
users (the condition which favors the better learning of users) to automatically personalize its learning environment.

In [14] the authors present a scheme that looks for the behaviors that are more significant towards identifying the learning styles of the LMS users, considering affective and performance patterns, for example "how many exercises did the user visit?", or "were these exercises basic, intermediate or advanced?".

In [1], the author investigates the perception, in terms of constructiveness, of undergraduate and graduate accounting students about the difference between: 1- Receiving timely feedback ( 5 days) against extremely timely feedback ( 2.5 days or even instant), and 2- Receiving manually written formal assessment feedback against automatically generated feedback.

In [7] the authors evaluate the role of e-assessments feedback through the creation of two study-groups of undergraduate engineering students. Both groups received teaching based on the same traditional way, enhanced with VLE components. The difference lied in the form of e-assessments and feedback given. Group 1 (CG) gave students just one opportunity of e-assessment, indicating only if each answer was correct or not. Group 2 (EG) gave students unlimited opportunities of e-assessments, giving clues as to why each incorrectly-answered question was incorrect. Students of Group 2 had an overall better performance.

\section{Feedback in Assessment}

Nicol \& Macfarland-Dick [9] established seven principles for good practices in feedback. These principles are related to: clarify what good performance is (goals, criteria and expected standards); facilitate the development of self-assessment (reflection) in learning; deliver high-quality information to students about their learning; encourage dialog between peer professors; encourage positive motivational beliefs; provide opportunities to close the gap between current and desired performance; and provide information to professors to help shape teaching. The authors of this work consider that four of the mentioned principles are present in the proposed feedback scheme:

1. It helps to clarify what good performance is (goals, criteria, expected standards).

The scheme would include features to develop some strategies, such as the following:

- The application of a questionnaire to the students (in the VLE) to find out to what extent they understand the objectives, criteria and evaluation standards.

- The offering of examples of performance (in the VLE). Examples are effective because they make explicit what is required and define valid standards for students to compare their work.

2. It facilitates the development of self-assessment (reflection) in learning.

The feedback scheme proposed should provide a sequence of simple questions to generate confidence in the student when performing the self-assessment 
and when looking at the evaluation given by the system; these questions may be repeated several times.

3. It delivers high-quality information to students about their learning.

Specifically, the scheme provides:

- Opportunistic feedback to allow students to correct their work while still learning a subject in the course.

- Corrective advice, which also indicates strengths and weakness.

- Online assessments and consequential feedback at any time, anywhere, and as many times as students wish.

The VLE should have assessments (and corresponding automatic feedback) available at all times to reinforce this good practice.

4. It provides opportunities to close the gap between current and desired performance.

Given a failure to answer correctly an MCQ, the scheme should offer the student indications of the possible reasons for that failure.

The next sub-section gives details of a scheme which, in order to offer feedback to students, captures their browsing.

\subsection{Complexity of Distractors}

An MCQ in the scheme would be composed of a question and four answers to choose from, which are: the right answer and three distractors. The distractors are classified in the following way:

1. Distractor 1 (D1). The most easy to discard; it requires the use of a single definition to realize that it is wrong.

2. Distractor 2 (D2). It requieres the use of analysis to discard, that is, it requires to combine data, obtained from the question, and concepts, to observe a contradiction.

3. Distractor 3 (D3). This is an answer that has a small mistake, it resembles to be the right one. It is, of course, the hardest one to elucidate to discard.

Each one of the distractors is associated with a browsing level in the feedback sequence (a feedback-level), and a level of error. Thus, D1, D2 and D3 are associated to Knowledge, Application and Test level, respectively. At next subsection feedback levels are explained.

\subsection{Feedback Levels}

This approach is conceived as a sequence of steps: from the explanation of why the student's response to an exercise was incorrect, to an example that explains how knowledge is used to solve this type of exercise, going through the concepts of the subtopic, related to the exercise. These would be the feedback-levels that the learning platform should provide, that would allow the registration of various variables in the comments offered. The navigation sequence should offer three feedback-levels: 
a) Knowledge level is a reminder about the implicit concepts of the exercise, where students can review each concept or continue with other related concept, if required. At the ending, students should be able to decide if they continue to solve more exercises or continue in the browsing sequence with an example that illustrates the application of concepts.

b) Application level shows the application of concepts in a simple exampleproblem, where there is always the possibility to visualize previous concepts, as many times as desired. In addition, the student should be able to change the example or to choose either to end, to continue with the exercises, or to move to other feedback-level.

c) Test level proposes students to solve an exercise related to the same subtopic, and with the same level of complexity as the one reviewed in the Application feedback-level, offering the opportunity to receive similar feedback if his answer was incorrect.

In the case of success, the student could return to do other exercises or move to any desired feedback-level, for the consolidation of learning.

\subsection{Example of a Screen Transit-Plan for the Assessment-Feedback Scheme}

The following list presents a transit-plan among the diverse screens of the assessmentfeedback scheme proposed. Figure 1 presents a diagram of the transit-plan.

A. A list of exercises appears on the screen.

B. The student chooses one exercise to solve.

C. The statement of the chosen exercise appears on the screen, together with a list of answers to choose from, among which only one answer is correct.

D. The student picks one answer (the student should really work without choosing randomly).

E. The scheme detects if the answer was correct or not. If it was incorrect, according to the answer the system classifies the level of error, to offer the student one of three feedback-levels.

F. This feedback-level presents an explanatory text oriented to the understanding of concepts.

G. This feedback-level presents an example with application of concepts.

H. This feedback-level presents a similar exercise to the one solved, and its solution-approach.

I. The scheme indicates that the solution of the problem was correct.

J. In case of having answered incorrectly, the student might choose to accept, or not, the feedback-levels offered given by the system, but, in any case, he can choose to transit to any one of the options A, F, G or H. 


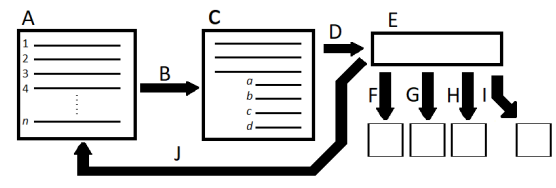

Fig. 1. Relations of the components of feedback in the VLE.

\subsection{Example of Feedback at MCQ Solution}

The topic of this example is 'Graph Theory', which is offered in the first year of the 'Information Technologies and Systems' undergraduate program. Among the following MCQ, items (a), (b) and (d) are distractors of type 3, 1, and 2, respectively.

If $\mathcal{G}^{\prime}=\left(\mathcal{V}^{\prime}, \mathcal{A}^{\prime}\right)$ is a subgraph of $\mathcal{G}=(\mathcal{V}, \mathcal{A})$ and $v \in \mathcal{V}^{\prime}$, then it is true that:

a) If $v$ is an isolated vertex in $\mathcal{G}^{\prime}$, then it is an isolated vertex in $\mathcal{G}$.

b) $\mathcal{G}^{\prime}$ is connected.

c) If there is a cycle $v-v$ in $\mathcal{G}^{\prime}$ then there is a simple cycle $v-v$ in $\mathcal{G}$.

d) There is a non-trivial cycle $v-v$ in $\mathcal{G}^{\prime}$.

Let us see how would the browsing scheme be. It is important to take into account that there are many previous concepts. The content of each one the three feedback-levels offered could be:

Knowledge Pay attention to the following definitions:

1. A connected graph must satisfy the definition: $A$ graph $\mathcal{G}=(\mathcal{V}, \mathcal{A})$ is connected if for all pair of vertices, $x, y \in \mathcal{V}$ there exists a path $x-y$.

2. A subgraph $\mathcal{G}^{\prime}$ is constructed from a given graph, $\mathcal{G}$, accordingly the definition: The graph $\mathcal{G}^{\prime}=\left(\mathcal{V}^{\prime}, \mathcal{A}^{\prime}\right)$ is a subgraph of $\mathcal{G}$, iff $\mathcal{V}^{\prime} \subset \mathcal{V}, \mathcal{A}^{\prime} \subset$ $\mathcal{A}$, and for every one of its edges a $\in \mathcal{A}^{\prime}$ its extremes hold $v_{1}, v_{2} \in \mathcal{V}^{\prime}$.

Application In order to build a subgraph we must choose $\mathcal{V}^{\prime}$ and $\mathcal{A}^{\prime}$. We can choose any subset of vertices from $\mathcal{V}$ to define $\mathcal{V}^{\prime}$.

For example, let $\mathcal{G}$ be given by the following graph:

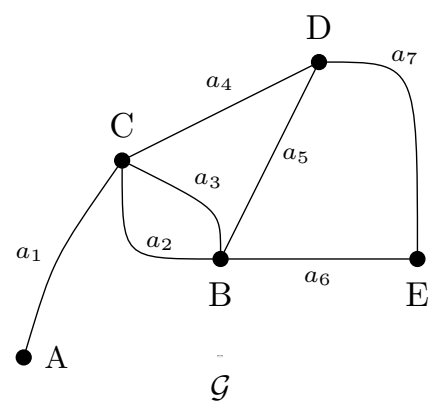


For instance, we can define $\mathcal{V}^{\prime}$ as $\{A, C, B, E\}$, or $\{E, D, A\}$, or also $\emptyset$. For the next step, we add the edges as we want. If we defined $\mathcal{V}^{\prime}$ as $\{A, C, D, B\}$, the edges of this subgraph could be a subset taken from $\left\{a_{1}, a_{2}, a_{3}, a_{4}, a_{5}\right\} \subset \mathcal{A}$. A choice might be $\left\{a_{1}, a_{3}, a_{5}\right\}$, or $\left\{a_{4}\right\}$ or $\emptyset$. For $\mathcal{V}^{\prime}$ we could build a subgraph $\mathcal{G}^{\prime}$ with edges $\left\{a_{1}, a_{3}, a_{5}\right\}$, or build another subgraph $\mathcal{G}^{\prime \prime}$ with edge $\left\{a_{4}\right\}$.
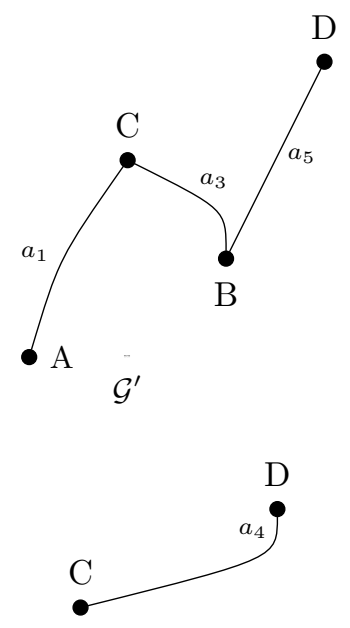

$\mathrm{B}$

- A

$\mathcal{G}^{\prime \prime}$

We remark that $\mathcal{G}^{\prime}$ is connected and $\mathcal{G}^{\prime \prime}$ does not.

Test For the graph $\mathcal{G}$, we now consider another subset $\mathcal{V}^{\prime \prime} \subset \mathcal{V}$, for example

$\{B, C, E\}$. Construct three subgraphs, two connected and one disconnected.

\section{Conclusions and Future Work}

This paper presented an informal operation specification of a proposed assessmentfeedback scheme, which would be part of the kind of VLEs which provide higher-education students with the possibility to solve assessments focused on solving problems with MCQs, and which offer feedback in relation to a possible wrong answer. In the case of an incorrect answer from a student, the complexity of having discarded options to choose that answer becomes the key to offering the student one of several feedback-levels. One level can present the student with important conceptual content for its study; another level can present the student with useful relationships of concepts; and another level can show the student examples with applications similar to the exercise answered erroneously. This design has basis on the principles of good feedback-practices in self-assessment. 
The main subsequent work would be to implement the proposed scheme in a VLE. Subsequently, it would be interesting to explore how students browse and what their regularities are, based on their behavior and their school performance. In addition, it would be important to broaden the scope of this proposal, for example, to have the possibility to, automatically, generate specific exploration spaces for a question from an ontology (a set of related concepts) of the same subject of the question. There is also interest in moving from assessments of MCQs, to assessments with open questions, which would require the application of natural language processing, for the generation of the related automaticfeedback with short answers.

Acknowledgments. This work was partially supported by the Department of Information Technologies of our university, UAM-C.

\section{References}

1. Bayerlein, L.: Students' feedback preferences: how do students react to timely and automatically generated assessment feedback? Assessment \& Evaluation in Higher Education, 39(8):916-931, Routledge, https://doi.org/10.1080/02602938.2013.870531 (2014)

2. Boud, D.: Sustainable assessment: rethinking assessment for the learning society. Studies in Continuing Education, 22(2):151-167 (2000)

3. Boud, D., Molloy, E.: Rethinking models of feedback for learning: The challenge of design, Assessment and Evaluation in Higher Education, 38(6):698-712 (2013).

4. Carless, D., Salter, D., Yang, M., Lam, J.: Developing sustainable feedback practices. Studies in Higher Education, 36(4), 395-407 (2011)

5. Gibbs, G.: Using assessment strategically to change the way students learn. In: Assessment Matters in Higher Education, Sally Brown \& Angela Glasner, editors. Buckingham: Society for Research into Higher Education and Open University Press (1999)

6. Hounsell, D.: Towards more sustainable feedback to students. In: Rethinking Assessment in Higher Education. Learning for the longer term, David Boud \& Nancy Falchikov, editors, pp. 101-113. Londres y Nueva York: Routledge (2007)

7. Limniou. M, Smith, M.: The role of feedbak in e-assessments for engineering education. Educatin and Information Technologies, 19:209-225 (2014)

8. Moreno, T.: Evaluación del aprendizaje y para el aprendizaje. Reinventar la evaluación en el aula. Ciudad de México: Universidad Autónoma MetropolitanaCuajimalpa (2016)

9. Nicol, D., Macfarlane-Dick, D.: Formative assessment and self-regulated learning: A model and seven principles of good feedback practice. Studies in Higher Education, 31(2):199-218 (2006)

10. Nicol, D., Thomson, A., Breslin, C.: Rethinking feedback practices in higher education: A peer review perspective. Assessment and Evaluation in Higher Education, 39(1):102-122 (2014)

11. Núñez, L., et al.: A framework for automatic identification of learning styles in learning management systems. Research in Computing Science, 106:59-68 (2015)

12. Sadler, R.: Formative assessment and the design of instructional systems. Instructional Science, 18:119-144 (1989) 
13. Sadler, R.: Formative assessment: revisiting the territory. Assessment in Education, $5(1): 77-84$ (1998)

14. Salazar, G., et al.: Behavioral patterns for automatic detection of learning styles in learning management systems: a case study. Research in Computing Science, 106:69-77 (2015)

15. Torrance, H., Pryor, J.: Investigating formative assessment. Buckingham, Open University Press (1998)

16. Yorke, M.: Formative assessment and its relevance to retention. Higher Education Research and Development, 20(2):115-126 (2001) 